\title{
Establishing an integrated human milk banking approach to strengthen newborn care
}

\author{
A DeMarchis ${ }^{1,2}$, K Israel-Ballard ${ }^{1}$, Kimberly Amundson Mansen ${ }^{1,3}$ and C Engmann ${ }^{1,3,4,5}$
}

The provision of donor human milk can significantly reduce morbidity and mortality among vulnerable infants and is recommended by the World Health Organization as the next best option when a mother's own milk is unavailable. Regulated human milk banks can meet this need, however, scale-up has been hindered by the absence of an appropriate model for resourcelimited settings and a lack of policy support for human milk banks and for the operational procedures supporting them. To reduce infant mortality, human milk banking systems need to be scaled up and integrated with other components of newborn care. This article draws on current guidelines and best practices from human milk banks to offer a compilation of universal requirements that provide a foundation for an integrated model of newborn care that is appropriate for low- and high-resource settings alike.

Journal of Perinatology (2017) 37, 469-474; doi:10.1038/jp.2016.198; published online 10 November 2016

\section{INTRODUCTION}

The value of breastfeeding (BF) and donated human milk (DHM) for providing optimal nutrition and immunological protection for infants is well established. ${ }^{1,2}$ Considered a pillar of child survival worldwide, human milk is both immunologically and nutritionally ideal for infants, ${ }^{3}$ and is especially important for infants born premature or in resource-limited settings. ${ }^{4-10}$ Yet many babies lack access to their mother's own milk (MOM) due to issues of abandonment, mother's death, baby's illness or inability to suckle, or delay in milk production. This lack of access leaves babies more vulnerable to disease, poor health or death. ${ }^{10-12}$

Providing DHM to vulnerable neonates without access to MOM not only directly saves lives but also increases awareness of the value of BF and improves overall community BF rates. ${ }^{10,13-15}$ This increase in $B F$ rates is important because suboptimal BF contributes to an estimated $11.6 \%$ of child deaths worldwide, or about 800000 deaths annually. ${ }^{16-18}$ When MOM is unavailable, the World Health Organization (WHO), ${ }^{19}$ American Academy of Pediatrics, ${ }^{20}$ European Society for Pediatric Gastroenterology, Hepatology, and Nutrition Committee on Nutrition, ${ }^{9}$ and other major policy groups recommend DHM as the next best option.

Historically, a human milk bank (HMB) is a service established to recruit human milk donors, collect DHM, and then process, screen, store and distribute the milk to meet infants' specific needs for optimal health. As part of the screening process, HMBs accept donations only from mothers who have more than enough milk to meet their own infant's needs. ${ }^{21-24}$ As an essential component of newborn care, an effective HMB system is a pre-requisite for ensuring infants' access to a safe, high-quality and sustainable supply of DHM when MOM is unavailable. Also, these systems have demonstrated substantial health benefits, especially for vulnerable babies. ${ }^{9,25}$

DHM programs can strengthen current practices in neonatal intensive care units (NICUs) to support BF by increasing the awareness of families and NICU staff of the value of BF for enhancing newborn health. ${ }^{14,15,26}$ For example, one study found that the exclusive BF rate for infants discharged from NICUs with an HMB (29.6\%) was significantly higher than the rate for infants from NICUs without an HMB (16.0\%). ${ }^{27}$ In another study, infants discharged 2 years after the start of a NICU HMB program had sixfold higher odds of receiving MOM at discharge as well as a $49 \%$ reduction in the cessation of MOM consumption during hospitalization as compared to infants before the start of the program. ${ }^{14}$ In addition to the benefits of HMBs in increasing BF rates, the provision of $\mathrm{DHM}$ as a nutrition intervention has beneficial effects on neurodevelopmental outcomes, risk of sepsis, risk of necrotizing enterocolitis, tolerance of feedings, length of stay in the NICU and direct cost savings. ${ }^{5,7-10,27-34}$

As the global community revitalizes efforts to reduce preventable newborn mortality and help babies thrive, ${ }^{19}$ the commitment to human milk banking has rapidly expanded. Yet the roughly $500 \mathrm{HMBs}$ operating worldwide follow varying systems of regulation and are integrated to varying degrees within local and national health-care systems. ${ }^{35}$ As a result, countries have had mixed results in implementing HMB systems. A lack of infrastructure, regulation, and guidance in many of these HMBs hinders quality control, safety, scale-up and generation of community BF support. In many countries, a shortage of HMBs; a lack of community, policy and financial support; and competition from breast milk substitutes and unregulated milk sharing have prevented DHM from reaching many vulnerable neonates. ${ }^{36}$

A key finding from previous experience is that HMBs thrive in countries where they are protected, promoted, and supported as a vital component of national BF policies and newborn care. ${ }^{37}$ Building off of PATH's existing toolkit for the establishment of HMBs, 'Strengthening Human Milk Banking: A Global Implementation Framework', ${ }^{35}$ the purpose of this document is to guide policy makers and health leaders who are developing HMB strategies for their countries and to help strengthen existing HMB systems around the world.

\footnotetext{
${ }^{1}$ Maternal, Newborn, Child Health and Nutrition Program PATH, Seattle, WA, USA; ${ }^{2}$ Nutritional Sciences Program, University of Washington, Seattle, WA, USA; ${ }^{3}$ Seattle Children's Hospital, Seattle, WA, USA; ${ }^{4}$ Department of Pediatrics, University of Washington, Seattle, WA, USA and ${ }^{5}$ Department of Global Health University of Washington, Seattle, WA, USA. Correspondence: Dr K Israel-Ballard, Maternal, Newborn, Child Health and Nutrition Program PATH, P.O. Box 900922, Seattle, WA 98109, USA.

E-mail: kisrael-ballard@path.org

Received 16 June 2016; revised 24 August 2016; accepted 30 August 2016; published online 10 November 2016
} 


\section{IDENTIFYING KEY FEATURES OF SUCCESSFUL PROGRAMS}

To identify key features of successful HMB programs and develop a model for effectively establishing and operating HMBs in lowresource settings, as outlined in 'Strengthening Human Milk Banking: A Global Implementation Framework', PATH systematically consulted the global HMB community; we called a Global Technical Advisory Group on HMB, visited numerous HMBs, and reviewed current guidelines and best practices from HMBs around the world, and consulted HMB implementers, neonatologists, nutrition experts and policy makers at the international, national and local levels. ${ }^{35}$ On the basis of this feedback, we developed a model outlining the steps needed to establish and operate an effective HMB system that meets the highest measures for quality control, sustainability and effectiveness. ${ }^{35}$

Our assessment found that comprehensively integrating the operation of HMBs within newborn care strategies, neonatal unit operational structures, BF support services and national and regional policies is crucial to success. ${ }^{35}$ This comprehensive approach to service provision enables HMBs to significantly expand their reach beyond the vulnerable neonates who need DHM and permits an even greater impact on infant health and newborn care. ${ }^{38}$ In addition to DHM collection, processing and storage in 'stand-alone' HMB models, an integrative approach

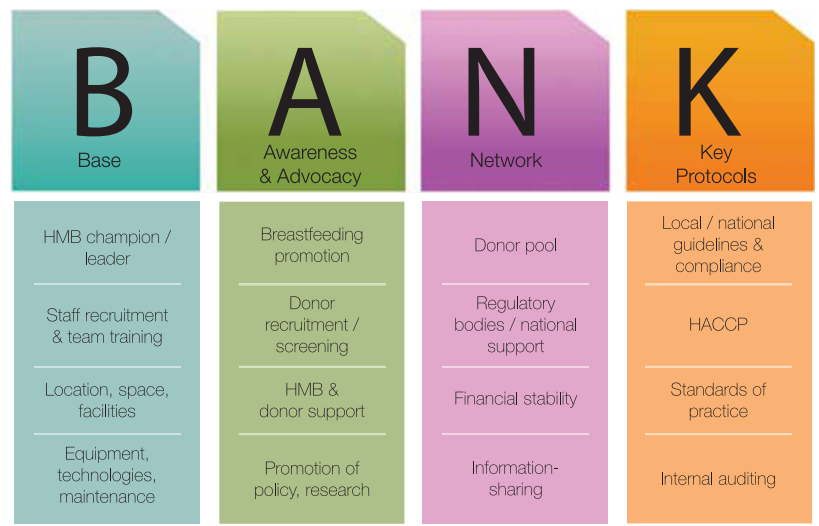

Figure 1. Four key components to establish an effective human milk bank: create a base (B), pursue awareness and advocacy (A), build a network $(\mathrm{N})$ and develop key protocols (K). HACCP, Hazard Analysis and Critical Control Points; $\mathrm{HMB}$, human milk bank. establishes HMBs as welcoming hubs within hospitals where all mothers, regardless of their desire to donate milk, can access support for BF and Kangaroo Mother Care, ${ }^{39-41}$ thus raising awareness of the importance of BF and markedly expanding the impact of HMBs. As natural centers of care for mothers and babies, this type of comprehensive HMB program can provide an active link to communities to improve neonatal health. Implemented this way, HMB activities create a self-reinforcing cycle of optimal BF practices to enhance infant health and improve newborn care. Because success requires attention to many facets of implementation, standardizing practices and providing guidance for core functions can help ensure a comprehensive, integrated and effective approach to human milk banking. In addition to outlining the key steps required to strengthen human milk banking, this document serves as a call to action for the global HMB community and policy makers to support this lifesaving intervention.

\section{A MODEL FOR ESTABLISHING HMBS}

Building successful, integrated HMB systems requires four key steps. Leaders must (1) establish the fundamental base necessary for quality assurance, and diverse expertise and ownership (for example, staff, space and tools); (2) pursue awareness of and advocacy for BF; (3) establish a network of HMBs to facilitate auditing, tracking, information sharing (that is, effective strategies) and partnerships; and (4) develop key protocols to guide operating procedures and the clinical provision of DHM (Figure 1).

\section{A base for quality assurance}

A solid foundation of personnel, facilities and equipment allows an $\mathrm{HMB}$ to employ the highest quality, safety and ethical practices for DHM collection, treatment and provision. This foundation also enables effective linkages to other essential newborn care interventions, such as BF promotion and Kangaroo Mother Care. Key personnel include an HMB champion, who is a clinical authority and hospital representative who can represent and advocate for the $\mathrm{HMB}$ while providing clinical expertise and operational oversight of HMB activities. Other HMB personnel and advocates may be drawn from a range of professional disciplines and positions found in hospitals and the local community. ${ }^{13,42,43}$ They must ensure adherence to handling and processing guidelines, maintain facilities, provide support to donor mothers and lactation support to BF mothers, and perform microbial testing of DHM. ${ }^{42,44}$ Table 1 outlines the positions and roles typically needed to operate an $\mathrm{HMB}$.

Table 1. Suggested roles for health-care personnel supporting a HMB

\begin{tabular}{|c|c|c|}
\hline $\begin{array}{l}\text { HMB operational team } \\
\text { structure }\end{array}$ & Skills/staffing & Roles and responsibilities \\
\hline HMB champion & Head neonatologist or pediatrician & $\begin{array}{l}\text { Provides overall supervision of the HMB quality } \\
\text { control, distribution and operations } \\
\text { Provides technical expertise }\end{array}$ \\
\hline $\begin{array}{l}\text { HMB advisory } \\
\text { committee }\end{array}$ & $\begin{array}{l}\text { Microbiologist, lactation support/advisor, HMB champion, community } \\
\text { leader, facility management, HMB technician, maternity operations } \\
\text { manager, nurse, dietitian and infection control }\end{array}$ & $\begin{array}{l}\text { Provides monitoring, feedback and guidance to } \\
\text { milk bank to ensure quality output and structure } \\
\text { Provides guidance on effective integration of core } \\
\text { HMB staff into hospital to support neonatal care } \\
\text { Advocates for HMB in local community }\end{array}$ \\
\hline Core HMB staff & Lactation support specialist and HMB technician & $\begin{array}{l}\text { Ensures lactation support is available to mothers } \\
\text { in HMB, ward and community } \\
\text { Oversees the handling and processing of donor } \\
\text { milk } \\
\text { Manages operations of HMB facility }\end{array}$ \\
\hline
\end{tabular}




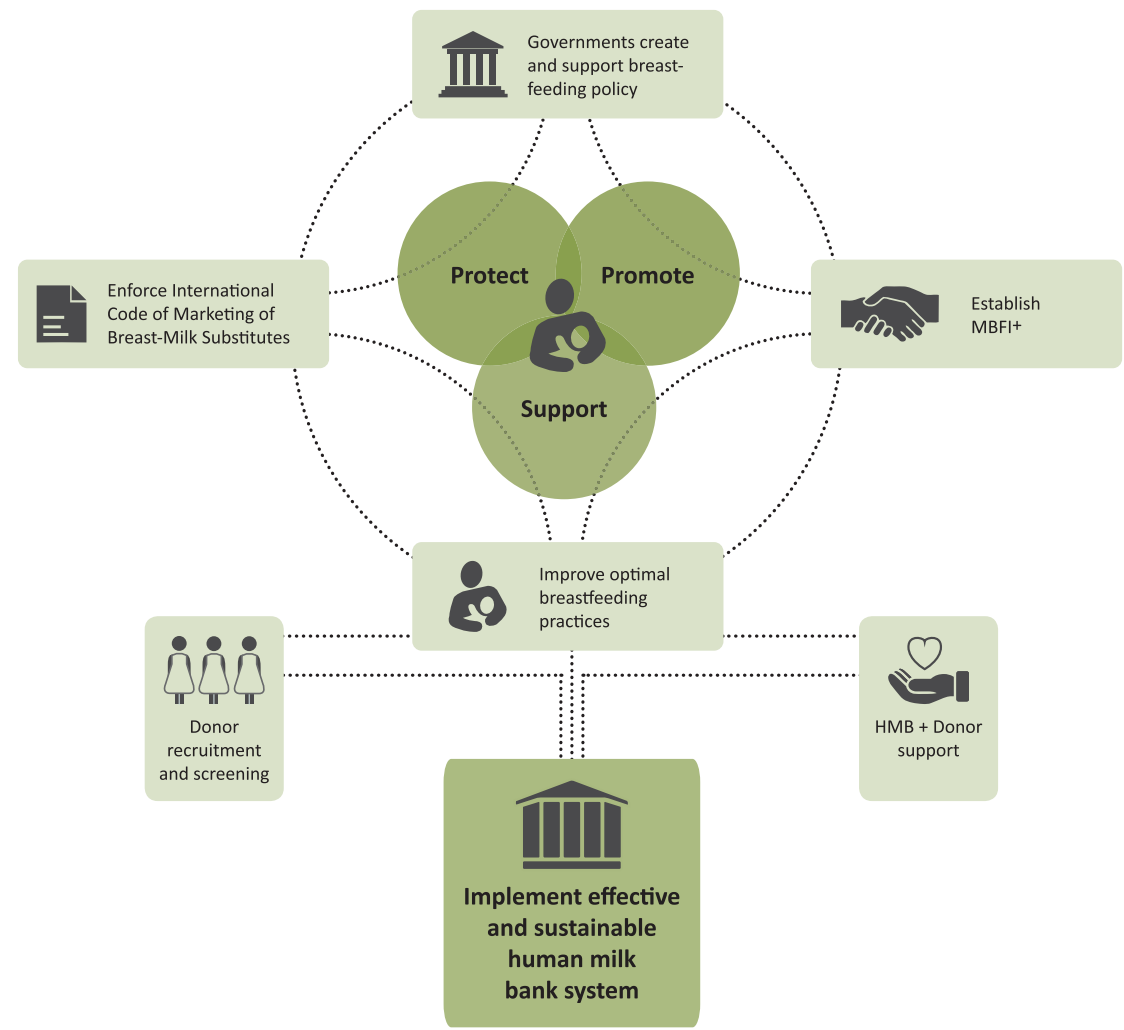

Figure 2. Overview of advocacy and awareness-raising activities to strengthen HMB systems. MBFI+ (Mother-Baby Friendly Initiative Plus) is a PATH-developed HMB model. It builds on the UNICEF MBFI approach with the added 'Plus' to incorporate HMB as part of a comprehensive package of breastfeeding support services.

Location within a hospital or proximity to a hospital can support operational procedures, increase convenience for milk donors and improve interactions with the neonatal care unit. ${ }^{15}$ Space and facilities affect HMBs' abilities to carry out operational procedures, and provide comfort and support to donors. ${ }^{43}$

In addition, HMBs require continual investment in high-quality, properly maintained equipment for the expression, storage and treatment of DHM, along with administrative processes and equipment for maintaining quality-control records, and tracking and tracing donor samples and milk processing data. ${ }^{42,45,46}$ In settings without a reliable source of electricity, the HMB may also need an alternative energy supply. ${ }^{45,46}$

Awareness and advocacy

To protect, promote and support BF for all mothers and their infants, HMBs must increase awareness of the importance of BF and DHM, and advocate for policy support (Figure 2). Supporting $\mathrm{BF}$ is the cornerstone of work to build sustainable, effective HMB systems. Comprehensive support for and promotion of exclusive $\mathrm{BF}$ helps to build a larger milk donor pool and strengthen the foundation for HMBs. ${ }^{47}$

Integrating the HMB system with existing hospital and community support leadership and advocacy can increase donor convenience and reduce barriers to donation. These barriers may include a lack of confidence in the milk supply and lack of awareness of need. ${ }^{48,49}$ Community engagement with local leaders and promotion in health-care facilities can also support donor recruitment, $22,31,50,51$ and hospitals and their staff can provide oral and written donor screening as well as serologic blood tests for infectious diseases such as HIV, hepatitis B and C, and syphilis, as well as Creutzfeldt-Jakob Disease..$^{21,22,42}$ To prevent negative impacts on donors' own children, the global practice of milk donation relies on volunteer donations only from mothers with milk in excess of their own infants' current and potential future needs. ${ }^{35}$

Policy support is also needed at the local, national and international levels. At each level, the provision of DHM through HMBs needs to be integrated into infant and young child nutrition, and newborn care policies and guidelines to achieve the best results. ${ }^{45,52-54}$

Finally, rigorous implementation science research is critical to further strengthen the evidence base for HMBs and exclusive BF. This research can provide the basis for advocacy, inform policy, ${ }^{9}$ and support consistent regulatory and operational procedures across countries and regulatory agencies.

\section{Networking}

Robust networks strengthen and support strategies for newborn care and HMB integration in several ways. For example, networks 'among' HMBs increase the quantity of available $\mathrm{DHM}^{55}$ help HMBs embrace best practices and create transparency in documentation of successful processes and results, which can support newborn care systems worldwide. ${ }^{56}$ Collaborative networks have been established in many regions to facilitate information sharing and self-regulation during the creation of network guidelines. ${ }^{35,37}$

In addition, strong relationships with milk donors help to build a stable, supportive community. ${ }^{57}$ Partnerships between HMBs and governmental agencies enable HMBs to provide assistance, and advice for improving and expanding national milk banking policies and guidelines, increase awareness of the need for HMBs, and support auditing and tracking procedures at the local 

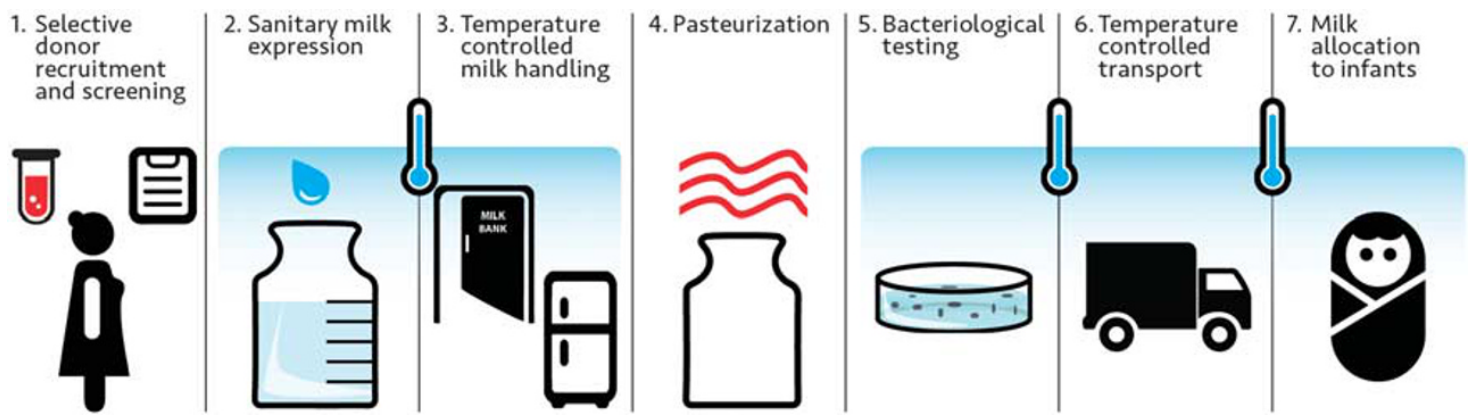

Figure 3. Procedures at human milk banks should align with best practices for newborn care and use a Hazard Analysis and Critical Control Points (HACCP) process and internal auditing at each step.

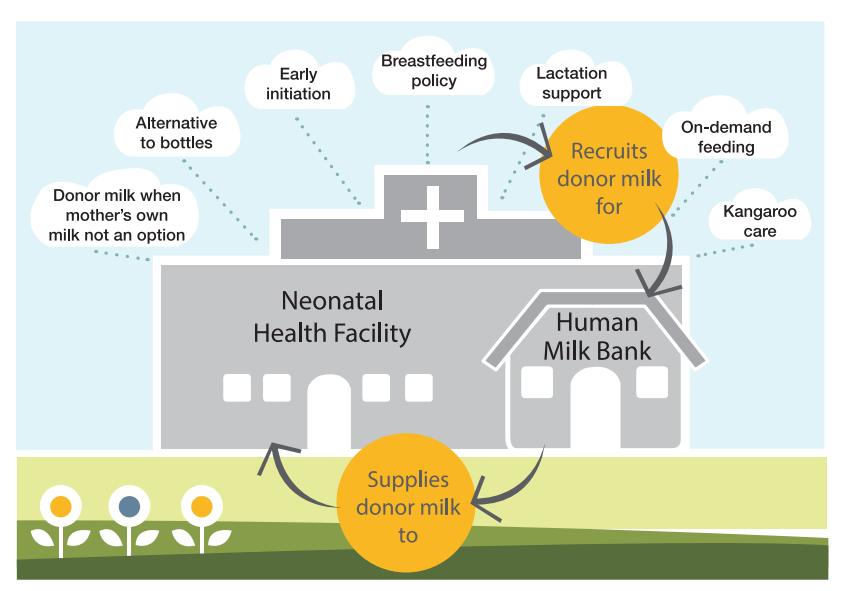

Figure 4. PATH's Mother-Baby Friendly Initiative Plus program, in which human milk banks have a critical role in supporting breastfeeding.

level. ${ }^{25,58,59}$ Financial supporters ease the burden of costs and provide support for investments and technology improvements. ${ }^{60}$ Connected professionals and experts share information and evidence, which is crucial to establishing strong systems. ${ }^{35,61}$ Innovative partnerships involving HMBs, employers, and community leaders promote and support BF (for example, through breaks to pump or breastfeed and refrigerators to store milk), build trust and support within the community, and create a powerful reinforcing cycle in which outreach, lactation support, BF promotion and HMBs continuously increase access to DHM.

\section{Key protocols}

Key protocols guide the standardization and operationalization of HMBs. They support the clinical provision of DHM and help to ensure adherence to rigorous quality-control methods.

Standards of practice should guide every step of DHM processing, from donor recruitment to delivery to infants. ${ }^{62,63}$ As one example, in the United States, the Human Milk Banking Association of North America has developed guidelines with support from the US Food and Drug Administration and the Centers for Disease Control and Prevention. ${ }^{24,46}$ In many other countries where HMBs already exist or are being planned, HMB guidelines still need to be developed to ensure the highest standards possible within the local context ${ }^{64,65}$ and to ensure alignment with local and global infant and young child nutrition and newborn care guidelines. Simple pasteurization methods can ensure quality standards of DHM in resource-limited settings without a reliable energy supply. ${ }^{66,67}$

Existing safety systems can be used to ensure quality standards for DHM. For example, the Hazard Analysis and Critical Control Points process can be considered a best practice for developing a locally adapted, rigorous safety analysis and action plan for the handling and treatment of DHM. ${ }^{63}$ Use of the Hazard Analysis and Critical Control Points process enables local technical experts to map out the process steps and identify points at which hazards, contamination or mishandling can occur, in their own, unique setting (Figure 3). ${ }^{68}$

Because guidelines are not enforced at the national level in some countries, ${ }^{58}$ HMBs must often self-regulate as an independent body or network to ensure proper practices and support for auditing. Internal auditing can improve systems of quality control. Detailed, periodically validated records also allow for immediate tracking and tracing if a rapid recall of DHM is needed. ${ }^{22}$

\section{DISCUSSION AND CONCLUSION}

When MOM is not available, DHM provides vulnerable infants with a new chance to thrive. ${ }^{65,69}$ According to the United Nations' 'International Covenant on Economics, Social and Cultural Rights and the Declaration and Convention on the Rights of the Child', access to a safe, high-quality supply of DHM is part of a child's human right to optimal nutrition. ${ }^{37}$ Effective implementation of $\mathrm{HMBs}$ is crucial to enable more infants around the world to receive the benefits of human milk.

Despite growing interest and investment in HMBs worldwide, fragmented systems and practices, a lack of alignment among HMBs, lack of government and policy support, and other factors often prevent HMBs from reaching their full potential. ${ }^{37}$ Systems of newborn care can be strengthened by integrating HMBs with health-care services and BF support, which will bolster the infrastructure, human resources, and technical and financial means necessary for strong milk banking systems and improve national strategies for reducing neonatal and infant mortality. An approach to strengthening HMBs that (1) establishes a strong base, (2) raises awareness and conducts advocacy, (3) creates a network and (4) develops key protocols will help integrate key components of a successful HMB system.

On the basis of the Brazil National Network of Human Milk Banks, PATH's Mother-Baby Friendly Initiative Plus model promotes a comprehensive BF package (Figure 4). ${ }^{12,35}$ With $>219$ HMBs nationally and outreach to $>23$ countries, the Brazilian network is the world's largest and most effective system of HMBs. The system extends human milk banking far beyond neonates in intensive care units. In 2015, > 170000 neonates received DHM 
and $>2$ million women received lactation support through HMBs in Brazil. ${ }^{70}$

Government has an especially important role in successfully integrating human milk banking with newborn care. When governments integrate HMBs with national infrastructures, support services, networks and guidelines, they are fulfilling an ethical responsibility to improve child health and reduce mortality by providing the best nutrition available to all children. The proven medical and social benefits of DHM contribute to achieving the health goals of national governments, the WHO, and other groups worldwide. In addition, HMBs are a cost-effective way for hospitals and governments to provide the highest level of care when MOM is not available. ${ }^{71,72}$ Brazil's integrative and comprehensive HMB approach is credited with saving an estimated US\$540 million annually in medical costs. ${ }^{37}$ It is in governments' long-term best interest-as well as in the interest of mothers, children and communities - to develop strategies that support an integrated $\mathrm{HMB}$ system and implement, monitor and evaluate comprehensive newborn health-care and BF policies to ensure that HMBs achieve maximum impact.

In conclusion, integrating HMB systems into the larger framework of newborn care services may significantly increase access to human milk (as DHM or MOM), increase the impact of HMBs along the continuum of infant health-care, and help milk banking systems meet the increasing worldwide demand for $\mathrm{DHM}^{35,73} \mathrm{~A}$ comprehensive $\mathrm{HMB}$ model should be integrated with other components of a newborn care package, including BF promotion and Kangaroo Mother Care; similar to the American Academy of Pediatrics Helping Babies Survive-Essential Care for Every Baby and Helping Babies Breathe, and various Infection Prevention models. It is vital to promote, extend and integrate the reach of current and future HMBs into other newborn best practices and packages so that safe and high-quality milk to infants always assured. Through this improved support for mothers and infants and a sustainable approach will be created for saving more infant lives through a reinforcing cycle of increased awareness of BF and increased overall BF rates in the community.

Regardless of geographic location or resource availability, HMB core requirements and quality principles are universal. Strong, integrated HMB systems require local, national and global support to align them with community needs and resources. With support, they can provide a successful, cost-effective and sustainable approach to strengthening newborn care and improving infant survival and health. ${ }^{35}$

Human milk banking is most successful in countries where there is policy support and cooperation between health authorities and the milk banking industry. Fostering environments conducive to HMBs through use of standardized quality-control guidelines, effective communication, evidence generation and policy change is thus an essential component of effective newborn care. ${ }^{37}$ Institutions with a keen interest in newborn and infant health can improve newborn access to human milk by increasing investments in HMBs as part of integrated BF, newborn care and infant nutrition programs. ${ }^{74}$ Governments and health authorities can have a crucial role in providing the best possible nutrition for vulnerable infants by supporting an integrated system of care that includes human milk banking.

\section{CONFLICT OF INTEREST}

The authors declare no conflict of interest.

\section{ACKNOWLEDGEMENTS}

We gratefully acknowledge the technical and policy experts around the world who provided guidance. Our special gratitude is extended to the PATH Global Technical Advisory Group on Human Milk Banking who convened in November 2012 and formed the basis of this work. PATH also acknowledges the more than 500 human milk banks worldwide who are dedicated to providing safe donor human milk and saving infants' lives.

\section{REFERENCES}

1 US Department of Health and Human Services. The Surgeon General's call to action to support breastfeeding. U.S. Department of Health and Human Services, Office of the Surgeon General: Washington, WA, USA, 2011.

2 WHO Collaborative Study Team on the role of breastfeeding on the prevention of infant mortality. Effect of breastfeeding on infant and child mortality due to infectious diseases in less developed countries: a pooled analysis. Lancet 2000; 355(9202): 451-455

3 Ballard O, Morrow AL. Human milk composition: nutrients and bioactive factors. Pediatr Clin North Am 2013; 60(1): 49-74.

4 Sisk PM, Lovelady CA, Dillard RG, Gruber KJ, O'Shea TM. Early human milk feeding is associated with a lower risk of necrotizing enterocolitis in very low birth weight infants. J Perinatol 2007; 27(7): 428-433.

5 Meinzen-Derr J, Poindexter B, Wrage L, Morrow AL, Stoll B, Donovan EF. Role of human milk in extremely low birth weight infants' risk of necrotizing enterocolitis or death. J Perinatol 2009; 29(1): 57-62.

6 Quigley M, Henderson G, Anthony M, McGuire W. Formula milk versus donor breast milk for feeding preterm or low birth weight infants. Cochrane Database Syst Rev 2011; 93(3): 81-86.

7 McGuire W, Anthony M. Donor human milk versus formula for preventing necrotising enterocolitis in preterm infants: systematic review. Arch Dis Child Fetal Neonatal Ed 2003; 88(1): F11-F14.

8 Boyd CA, Quigley MA, Brocklehurst P. Donor breast milk versus infant formula for preterm infants: systematic review and meta-analysis. Arch Dis Child Fetal Neonatal Ed 2007; 92(3): F169-F175.

9 Arslanoglu S, Corpeleijn W, Moro G, Braegger C, Campoy C, Colomb V et al. Donor human milk for preterm infants: current evidence and research directions. J Pediatr Gastroenterol Nutr 2013; 57(4): 535-542.

10 Bertino E, Giuliani F, Baricco M, Di Nicola P, Peila C, Vassia C et al. Benefits of donor milk in the feeding of preterm infants. Early Hum Dev 2013; 89: S3-S6.

11 Panczuk J, Unger S, O'Connor D, Lee SK. Human donor milk for the vulnerable infant: a Canadian perspective. Int Breastfeed J 2014; 9: 4.

12 Tully MR, Lockhart-Borman L, Updegrove K. Stories of success: the use of donor milk is increasing in North America. J Hum Lact 2004; 20(1): 75-77.

13 Fugate K, Hernandez I, Ashmeade T, Miladinovic B, Spatz DL. Improving human milk and breastfeeding practices in the NICU. J Obstet Gynecol Neonatal Nurs 2015; 44(3): 426-438.

14 Parker MG, Burnham L, Mao W, Philipp BL, Merewood A. Implementation of a Donor Milk Program is associated with greater consumption of mothers' own milk among VLBW infants in a US, Level 3 NICU. J Hum Lact 2016; 32(2): 221-228.

15 Vázquez-Román S, Bustos-Lozano G, López-Maestro M, Rodríguez-López J, OrbeaGallardo C, Samaniego-Fernández M et al. Clinical impact of opening a human milk bank in a neonatal unit. An Pediatr 2014; 81(3): 155-160.

16 Black RE, Victora CG, Walker SP, Bhutta ZA, Christian P, de Onis M et al. Maternal and child undernutrition and overweight in low-income and middle-income countries. Lancet 2013; 382(9890): 427-451.

17 Black RE, Allen LH, Bhutta ZA, Caulfield LE, de Onis M, Ezzati M et al. Maternal and child undernutrition: global and regional exposures and health consequences. Lancet 2008; 371(9608): 243-260.

18 Bhutta ZA, Black RE. Global maternal, newborn, and child health-so near and yet so far. N Engl J Med 2013; 369(23): 2226-2235.

19 World Health Organization, United Nations Children's Fund. Every Newborn: an action plan to end preventable deaths. World Health Organization: Geneva, Switzerland, 2014.

20 Eidelman Al, Schanler RJ, Johnston M, Landers S, Noble L, Szucs K et al. Breastfeeding and the use of human milk. Pediatrics 2012; 129(3): e827-e841.

21 Arslanoglu S, Bertino E, Tonetto P, De Nisi G, Ambruzzi AM, Biasini A et al. Guidelines for the establishment and operation of a donor human milk bank: Italian Association of Human Milk Banks Associazione Italiana Banche del Latte Umano Donato (AIBLUD: www. aiblud. org). J Matern Fetal Neonatal Med 2010; 23 (S2): 1-20.

22 National Institute for Health and Clinical Excellence. Donor breast milk banks: the operation of donor breast milk bank services. National Institute for Health and Clinical Excellence: London, UK, 2010.

23 Grøvslien AH, Grønn M. Donor milk banking and breastfeeding in Norway. $J$ Hum Lact 2009; 25(2): 206-210.

24 Human Milk Banking Association of North America. Best Practice for Expressing, Storing and Handling Human Milk, 3rd edition. Fort Worth: TX, USA, 2011.

25 Mizuno K, Sakurai M, Itabashi K. Necessity of human milk banking in Japan: questionnaire survey of neonatologists. Pediatr Int 2015; 57(4): 639-644. 
26 Bertino E. IX. Effect of a human milk bank on breast-feeding rate in very-low-birthweight infants. J Pediatr Gastroenterol Nutr 2015; 61: S12.

27 Arslanoglu S, Moro GE, Bellu R, Turoli D, De Nisi G, Tonetto P et al. Presence of human milk bank is associated with elevated rate of exclusive breastfeeding in VLBW infants. J Perinat Med 2013; 41(2): 129-131.

28 Arnold LD. The cost-effectiveness of using banked donor milk in the neonatal intensive care unit: prevention of necrotizing enterocolitis. J Hum Lact 2002; 18(2): 172-177.

29 Isaacs EB, Fischl BR, Quinn BT, Chong WK, Gadian DG, Lucas A. Impact of breast milk on intelligence quotient, brain size, and white matter development. Pediatr Res 2010; 67(4): 357-362.

30 Rønnestad A, Abrahamsen TG, Medbø S, Reigstad H, Lossius K, Kaaresen Pl et al. Late-onset septicemia in a Norwegian national cohort of extremely premature infants receiving very early full human milk feeding. Pediatrics 2005; 115(3): e269-e276.

31 Updegrove K. Nonprofit human milk banking in the United States. J Midwifery Womens Health 2013; 58(5): 502-508.

32 Wight NE. Donor human milk for preterm infants. J Perinatol 2001; 21(4): 249-254.

33 Ganapathy V, Hay JW, Kim JH. Costs of necrotizing enterocolitis and costeffectiveness of exclusively human milk-based products in feeding extremely premature infants. Breastfeed Med 2012; 7(1): 29-37.

34 Schanler RJ, Shulman RJ, Lau C. Feeding strategies for premature infants: beneficial outcomes of feeding fortified human milk versus preterm formula. Pediatrics 1999; 103(6): 1150-1157.

35 PATH. Strengthening Human Milk Banking: A Global Implementation Framework. Version 1 Bill \& Melinda Gates Foundation Grand Challenges initiative, PATH: Seattle, WA, USA, 2013

36 Jones F. Milk sharing: how it undermines breastfeeding. Breastfeed Rev 2013; 21 (3): 21.

37 Arnold LD. Global health policies that support the use of banked donor human milk: a human rights issue. Int Breastfeed J 2006; 1: 26.

$38 \mathrm{Kim} \mathrm{JH}$, Chan CS, Vaucher YE, Stellwagen LM. Challenges in the practice of human milk nutrition in the neonatal intensive care unit. Early Hum Dev 2013; 89(Suppl 2): S35-S38.

39 Seidman G, Unnikrishnan S, Kenny E, Myslinski S, Cairns-Smith S, Mulligan B et al. Barriers and enablers of kangaroo mother care practice: a systematic review. PLoS One 2015; 10(5): e0125643.

40 Engmann C, Wall S, Darmstadt G, Valsangkar B, Claeson M. Consensus on kangaroo mother care acceleration. Lancet 2013; 382(9907): e26-e27.

41 Chaparro C, Lutter C. Beyond Survival: Integrated delivery care practices for longterm maternal and infant nutrition, health and development. Pan American Health Organization: Washington DC, WA, USA, 2007.

42 Human Milk Banking Association of North America. Guidelines for the Establishment and Operation of a Donor Human Milk Bank. Fort Worth: TX, USA, 2015.

43 Hurst NM, Myatt A, Schanler RJ. Growth and development of a hospital-based lactation program and mother's own milk bank. J Obstet Gynecol Neonatal Nurs 1998; 27(5): 503-510.

44 Renfrew MJ, Dyson L, McCormick F, Misso K, Stenhouse E, King S et al. Breastfeeding promotion for infants in neonatal units: a systematic review. Child Care Health Dev 2010; 36(2): 165-178.

45 Centers for Disease Control and Prevention. Proper Handeling and Storage of Human Milk. 2010. Available from http://www.cdc.gov/breastfeeding/recommen dations/handling_breastmilk.htm (accessed on 20 January 2015).

46 Robbins ST, Meyers R. Infant Feedings: Guidelines for Preparation of Human Milk and Formula in Health Care Facilities, 2nd ed. American Dietetic Association: Chicago, IL, USA, 2011.

47 Edwards TM, Spatz DL. Making the case for using donor human milk in vulnerable infants. Adv Neonatal Care 2012; 12(5): 273-278.

48 Thomaz ACP, Loureiro LVM, da Silva Oliveira T, NC Furtado Montenegro, Júnior EDA, Soriano CFR et al. The human milk donation experience: motives, influencing factors, and regular donation. $J$ Hum Lact 2008; 24(1): 69-76.

49 Kobler K. NICU bereavement breast milk donation (756). J Pain Symptom Manage 2012; 43(2): 453-454.

50 Azema E, Callahan S. Breast milk donors in France: a portrait of the typical donor and the utility of milk banking in the French breastfeeding context. J Hum Lact 2003; 19(2): 199-202.

51 Wilson-Clay B. The milk of human kindness: the story of the Mothers Milk Bank at Austin. Int Breastfeeding J 2006; 1(6): 1.

52 Gutierrez D, De Almeida J. Human milk banks in Brazil. J Hum Lact 1998; 14(4): 333.
53 The Innocenti Declaration at the WHO/UNICEF policymakers' meeting on. "Breastfeeding in the 1990s: A Global Initiative, held at the Spedale degli Innocent, $i$ " 1990.

54 World Health Organization and UNICEF. Baby-Friendly Hospital Initiative: Revised, updated and expanded for integrated care. Geneva, Switzerland, 2009.

55 Geraghty SR, List BA, Morrow GB. Guidelines for establishing a donor human milk depot. J Hum Lact 2010; 26(1): 49-52.

56 Brownell EA, Lussier MM, Herson VC, Hagadorn Jl, Marinelli KA. Donor human milk bank data collection in north america: an assessment of current status and future needs. J Hum Lact 2014; 30(1): 47-53.

57 Stevens J, Keim SA. How research on charitable giving can inform strategies to promote human milk donations to milk banks. J Hum Lact 2015; 31(3): 344-347.

58 Arnold LD. US health policy and access to banked donor human milk. Breastfeed Med 2008; 3(4): 221-229.

59 Marinelli KA, Lussier MM, Brownell E, Herson VC, Hagadorn Jl. The effect of a donor milk policy on the diet of very low birth weight infants. J Hum Lact 2014; 30 (3): $310-316$

60 Rosenbaum K. Implementing the use of donor milk in the hospital setting. Nurs Womens Health 2012; 16(3): 202-208.

61 Brownell KD, Roberto CA. Strategic science with policy impact. Lancet 2015; 385 (9986): 2445-2446.

62 Arnold L. Using banked donor milk in clinical settings. Reclaiming Breastfeeding for the United States: Protection, Promotion and Support. Jones and Barlett Publishers: Sudbury, MA, USA, 2002; 161-178.

63 Hartmann B, Pang W, Keil A, Hartmann P, Simmer K. Best practice guidelines for the operation of a donor human milk bank in an Australian NICU. Early Hum Dev 2007; 83(10): 667-673.

64 Tully MR. Recipient prioritization and use of human milk in the hospital setting. J Hum Lact 2002; 18(4): 393-396.

65 Arslanoglu S, Ziegler EE, Moro GE. WAPM Working Group on Nutrition. Donor human milk in preterm infant feeding: evidence and recommendations. $J$ Perinat Med 2010; 38(4): 347-351.

66 Chantry CJ, Wiedeman J, Buehring G, Peerson JM, Hayfron K, K'Aluoch O et al. Effect of flash-heat treatment on antimicrobial activity of breastmilk. Breastfeed Med 2011; 6(3): 111-116.

67 Naicker M, Coutsoudis A, Israel-Ballard K, Chaudhri R, Perin N, Mlisana K. Demonstrating the efficacy of the foneastra pasteurization monitor for human milk pasteurization in resource-limited settings. Breastfeed Med 2015; 10(2): 107-112.

68 Landers S, Hartmann BT. Donor human milk banking and the emergence of milk sharing. Pediatr Clin North Am 2013; 60(1): 247-260.

69 World Health Organization. Guidelines on optimal feeding of low birth-weight infants in low-and middle-income countries. World Health Organization: Geneva, Switzerland, 2011.

70 Rede Brasileira de Bancos de Leite Humano. A Rede Brasileira de Bancos de Leite Humano: Dados Estatistico. Rio de Janeiro, Brazil; 2015. Available from http:// www.redeblh.fiocruz.br/cgi/cgilua.exe/sys/start.htm?tpl = home (accessed on 18 August 2016).

71 Assad M, Elliott M, Abraham J. Decreased cost and improved feeding tolerance in VLBW infants fed an exclusive human milk diet. J Perinatol 2015; 36(3): 216-220.

72 Johnson TJ, Patel A, Bigger H, Engstrom J, Meier P. Cost savings of human milk as a strategy to reduce the incidence of necrotizing enterocolitis in very low birth weight infants. Neonatology 2015; 107(4): 271-276.

73 Perrine CG, Scanlon KS. Prevalence of use of human milk in US advanced care neonatal units. Pediatrics 2013; 131(6): 1066-1071.

74 Breastfeeding Innovations Team. Call to action: Expanding Access to Breastmilk: Banking on our most precious resource for babies. World Breastfeeding Week August 1-7, 2015. Available from http://www.europeanmilkbanking.com/images/ news/HumanMilkBanksCalltoAction2015.pdf (last date accessed 25 September 2015).

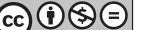

This work is licensed under a Creative Commons AttributionNonCommercial-NoDerivs 4.0 International License. The images or other third party material in this article are included in the article's Creative Commons license, unless indicated otherwise in the credit line; if the material is not included under the Creative Commons license, users will need to obtain permission from the license holder to reproduce the material. To view a copy of this license, visit http:// creativecommons.org/licenses/by-nc-nd/4.0/

(c) The Author(s) 2017 